EPJ Web of Conferences 47, 03003 (2013)

DOI: $10.1051 /$ epjconf/20134703003

(C) Owned by the authors, published by EDP Sciences, 2013

\title{
The Transit Monitoring in the South (TraMoS) project
}

\author{
Sergio Hoyer ${ }^{1,2 . a}$, Patricio Rojo ${ }^{3}$ and Mercedes López-Morales ${ }^{4}$ \\ ${ }^{1}$ Instituto de Astrofísica de Canarias, Via Láctea s/n, 38200 La Laguna, Tenerife, \\ Canary Islands, Spain \\ 2 Department of Astrophysics, University of La Laguna, via Láctea s/n, 38200 La Laguna, \\ Tenerife, Canary Islands, Spain \\ 3 Astronomy Department, Universidad de Chile, Casilla 36-D, Santiago de Chile, Chile \\ 4 Harvard-Smithsonian Center for Astrophysics, 60 Garden Street, Cambridge, \\ MA 02138, USA
}

\begin{abstract}
We present the Transit Monitoring in the South $(\operatorname{Tra} M o S)$ project. $\operatorname{Tra} M o S$ has monitored transits of 30 exoplanets with telescopes located in Chile since 2008, whit the following goals: (1) to refine the physical and/or orbital parameters of those exoplanet system, and (2) to search for variations in the midtimes of the transits and in other parameters such as orbital inclination or transit's depth, that could indicate the presence of additional bodies in the system. We highlight here the first results of $\operatorname{Tra} M \mathrm{oS}$ in three selected exoplanets.
\end{abstract}

\section{INTRODUCTION}

Almost 300 objects have been confirmed as transiting planets to date ${ }^{1}$ which more than $30 \%$ can be observed from southern facilities. This number is rapidly growing due to the several dedicated surveys to detect transiting exoplanets from ground-based telescopes located, in particular, in the Southern hemisphere (e.g. SuperWASP-South and HATSouth Network among others).

In the last years, the Transit Timing Variations (TTVs) technique [1-3] has been used to detect/confirm companions in the transiting system using the central time of the transits to measure the deviations from a constant orbital period [4-7]. In addition to the deviations in the mid-times of the transits, the gravitational interactions between these exoplanets can also induce changes in the inclination of the orbit and/or the measure of the ratio between the transiting exoplanet and star radii. All of these parameters can be extracted directly from the light curves during the transit [8].

The monitoring of exoplanetary transits through several epochs allows to determine with high accuracy the values of these parameters and to detect, if exist, variations induced by additional bodies in the system.

Since 2008, we started an observational campaign to monitor (in a short- and long term) the transits of known exoplanets from Southern facilities. In Section 2, we give a general description of the $T \mathrm{ra} M \mathrm{o} S$ project and in Section 3 we present a summary of the first published/submitted results.

\footnotetext{
ae-mail: shoyer@iac.es

1 exoplanets.eu
}

This is an Open Access article distributed under the terms of the Creative Commons Attribution License 2.0, which permits unrestricted use, distribution, and reproduction in any medium, provided the original work is properly cited. 


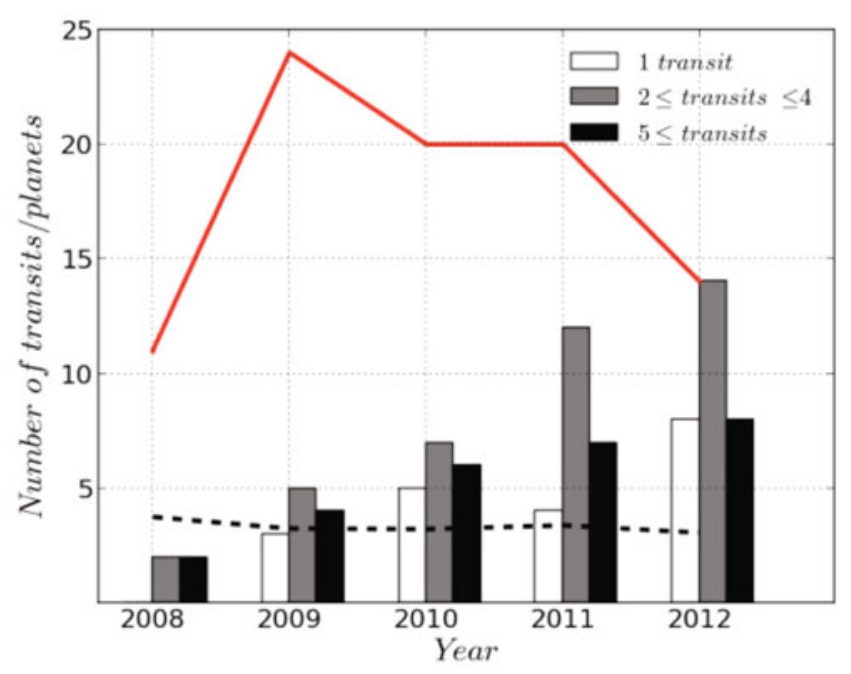

Figure 1. Statistics of the $\operatorname{Tra} M \mathrm{o} S$ project. The red solid line shows the number of transits observed per year by $T \mathrm{ra} M \mathrm{o} S$. The black dashed line represents the average of observed transits per planet per year. The white, gray and black bars represents the accumulative number of planets with 1, between 2 and 4, and with more than 5 transits observations by $\operatorname{Tra} M \mathrm{o} S$ since 2008 .

\section{TRAMOS PROJECT}

Since 2008, in the framework of the Transit Monitoring in the South project, we started to observe transits of already announced exoplanets using telescopes located in Chile. We use high cadence observations (30 to 50 seconds) in order to determine the central times of each transit with enough precision to detect departures from a constant keplerian orbit. Variations in other transit parameters can also be detected and the accumulative light curves will also refine the physical parameters of the transiting system. We use the new light curves presented by $\operatorname{Tra} M \mathrm{o} S$ in addition with the data available in the literature to perform an homogeneous analysis of them. Usually the light curves have different sources. Nowadays is not difficult to find a large number of light curves reported by several authors and therefore to perform an homogeneous analysis of the data, i.e., estimations of the correlated and noncorrelated noise (red or white noise), detrending, homogeneous assessment of uncertainties are critical in order to search for short and/or long term variations in the light curve parameters. In the same way, the use of all the available data for each system allows to obtain very precise values of global parameters for the system, such as the planet and star radius, the orbital inclination, ephemeris equation, etc.

\subsection{Observations}

For the monitoring of the transits we have used different diameter size telescopes ranging from 0.6-m to 8-m class telescopes. For relative bright targets (most of them WASP exoplanets) we have obtained data from the 0.6-m Southeastern Association for Research in Astronomy telescope (SARA-South) and the 1-m SMART telescope, both located in Cerro Tololo Inter-American Observatory (CTIO). We have also observations from the 4.1-m Southern Astrophysical Research (SOAR) at Cerro Pachón Observatory. For faint targets observations (mainly OGLE exoplanets) we take advantage of the large aperture of the Very Large Telescope (VLT) of the European Southern Observatory (ESO) and the Gemini-South Telescope at Cerro Pachón Observatory.

To date, almost 90 transits of 30 exoplanets have been observed by $T \mathrm{raMoS}$. In Fig. 1 we show a summary of the statistics (non-updated) of the $\operatorname{Tra} M o S$ project. The red solid line corresponds to the 


\section{Hot Planets and Cool Stars}

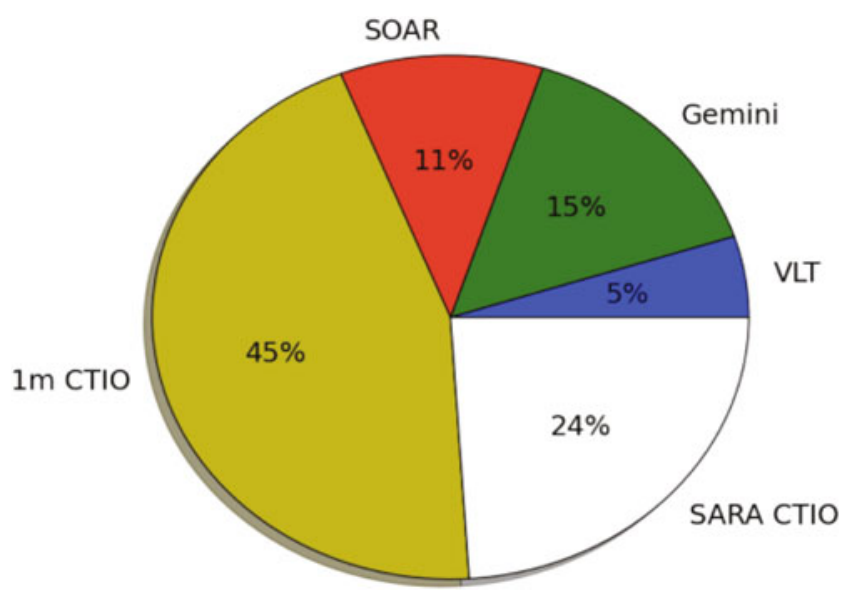

Figure 2. Percentage of transit observations from each telescope used by $\operatorname{Tra} M o S$.

number of transits observed each year since 2008. On average (represented by the dashed line), we have observed more than 3 transits per planet in our list. In Fig. 1 we also show the accumulative number of planets with only one transit observed (white bars), between two and four (gray bars) transits and with 5 or more (black bars) transit observations. In Fig. 2 we show the fraction of use of each telescope for our program.

\section{FIRST RESULTS}

We summarize the first results of the $\operatorname{Tra} M o S$ project based on the monitoring of three exoplanets: OGLE-TR-111b, WASP-5b and WASP-4b. With the TraMoS data we extended or complemented the time spam of the transit observations already reported for these systems. By this way we performed homogeneous analysis of all available data for each exoplanet.

\subsection{OGLE-TR-111b}

We observed five transits of OGLE-TR-111b using FORS1 and FORS2 at the ESO Very Large Telescope. Based on the transit mid-times of the new light curves we presented and together with the timing from the re-analysis of eleven previously published transits we confirmed that there is no significant evidence of TTVs since we found no variations larger than 1.5 minutes [9]. We derived upper limits in the mass of hypothetical companions of $1.3,0.4$ and $0.5 M_{\oplus}$ at the $3: 2,1: 2$, and $2: 1$ orbital resonances, respectively. These values are more strict than the limits imposed by the radial velocities technique.

\subsection{WASP-5b}

We reported nine new transit epochs of the Hot Jupiter WASP-5b [10]. These transits were observed in the I-band using the 1-m SMART telescope at CTIO and the 4.1-m SOAR telescope at Cerro Pachón Observatory. We combined the $\operatorname{Tra} M \mathrm{o} S$ light curves with eleven transits available in the literature to perform an homogeneous modelling and analysis of this system. For this exoplanet we found no TTVs rms variations larger than 60 seconds over 3 year time span (Fig. 3). We reject TTVs for this exoplanet since a constant orbital period can fully reproduce the obtained transit midtimes. Based on dynamical simulations of the WASP-5 system and the results of the timing analysis we discarded the presence of 


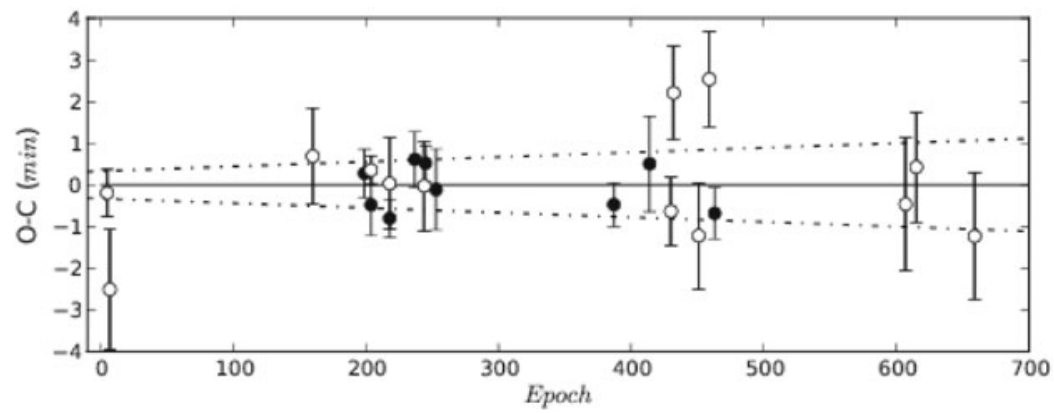

Figure 3. The Observed minus Calculated diagram of the transit midtimes of WASP-5b. Solid and open symbols correspond to the epochs reported by TraMoS [10] and by other authors, respectively. After an updated of the ephemeris equation the rms of the timing residuals is $\sim 1$ minute and all the transits do not deviate from a constant orbital period by more than $1-2 \sigma$ (represented by the dashed lines).

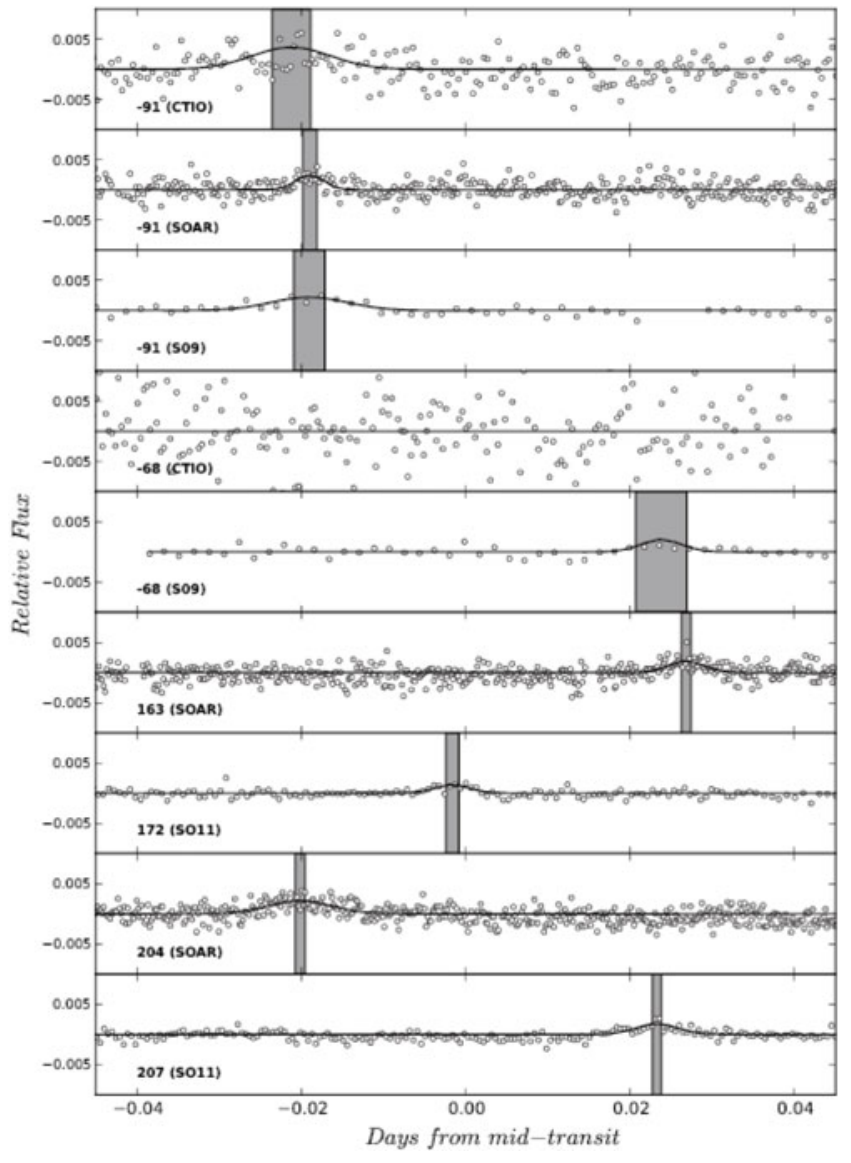

Figure 4. Star spot occultations are identified as bumps in the light curves during the transits of WASP-5b (figure from Hoyer et al. 2013). Solid lines correspond to the gaussian function fitted to the bumps. Gray regions are proportional to the errors in the timing of the occultation. The epoch and author of the light curve are indicated in the bottom left of each panel. See [11] (submitted) for details. 


\section{Hot Planets and Cool Stars}

additional planets with masses larger than 5, 1 and $2 M_{\oplus}$ around the 1:2, 5:3 and 2:1 orbital resonances with the transiting planet, respectively.

\subsection{WASP-4b}

We presented twelve epochs of the WASP-4b exoplanet [11, Submitted]. By performing an homogeneous analysis of the new light curves with the data available in the literature (twenty-six transits) we obtained individual values for the central time of the transits, the ratio of the planet and star radii and the orbital inclination for each transit. We found no evidence of the presence of more bodies in the system. We determined the most precise ephemeris equation at this time for this exoplanet. We also use the combination of the thirty-eight light curves to obtain very precise values of WASP-5b's physical parameters. During the transits we detected anomalies in the light curves that we identified as star spots occultations by the planet (Fig. 4). Based on the timing of these occultations we estimated the star rotational period is about 34 days, but additional observations of more consecutive epochs will allow to give better constraints.

\section{SUMMARY}

We presented the Transit Monitoring in the South (TraMoS) project which started in 2008 to observe transits of known exoplanets. To date almost 90 transits of 29 exoplanets have been observed. We highlighted in this contribution the first results of TraMoS. New data of this project are under analysis and will be presented soon. The monitoring of the transits have proven to be critical in order to determine physical parameters of the exoplanets and its host stars. Also, via timing analysis, this type of project provide strong constraints in the architecture of transiting systems. Results of Hot Jupiter transit monitoring have contributed to confirm the lack of low mass companions for this type of planet.

\section{References}

[1] J. Miralda-Escudé, ApJ 564, 1019 (2002), arXiv : astro-ph/0104034

[2] M.J. Holman, N.W. Murray, Science 307, 1288 (2005)

[3] E. Agol, J. Steffen, R. Sari, W. Clarkson, MNRAS 359, 567 (2005), arXiv : astro-ph/0412032

[4] M.J. Holman, D.C. Fabrycky, D. Ragozzine, E.B. Ford, J.H. Steffen, W.F. Welsh, J.J. Lissauer, D.W. Latham, G.W. Marcy, L.M. Walkowicz et al., Science 330, 51 (2010)

[5] W.D. Cochran, D.C. Fabrycky, G. Torres, F. Fressin, J.M. Désert, D. Ragozzine, D. Sasselov, J.J. Fortney, J.F. Rowe, E.J. Brugamyer et al., ApJS 197, 7 (2011), 1110.0820

[6] S. Ballard, D. Fabrycky, F. Fressin, D. Charbonneau, J.M. Desert, G. Torres, G. Marcy, C.J. Burke, H. Isaacson, C. Henze et al., ApJ 743, 200 (2011), 1109. 1561

[7] J.J. Lissauer, D.C. Fabrycky, E.B. Ford, W.J. Borucki, F. Fressin, G.W. Marcy, J.A. Orosz, J.F. Rowe, G. Torres, W.F. Welsh et al., Nature 470, 53 (2011), 1102.0291

[8] J.N. Winn, Exoplanet Transits and Occultations (2011), pp. 55-77

[9] S. Hoyer, P. Rojo, M. López-Morales, R.F. Díaz, J. Chambers, D. Minniti, ApJ 733, 53 (2011), 1102.3470

[10] S. Hoyer, P. Rojo, M. López-Morales, ApJ 748, 22 (2012), 1201.3616

[11] S. Hoyer, M. López-Morales, P. Rojo, MNRAS (2013) 